\title{
Traduire
}

Une autre perspective sur r tr traduction

Revue française de la traduction

$243 \mid 2020$

Les arts du spectacle

\section{Un théâtre qui traduit? Pistes de réflexion sur la théâtralité de la traduction}

\section{Antoine Palévody}

\section{(2) OpenEdition}

1 Journals

\section{Édition électronique}

URL : http://journals.openedition.org/traduire/2127

DOI : 10.4000/traduire.2127

ISSN : 2272-9992

Éditeur

Société française des traducteurs

Édition imprimée

Date de publication : 15 décembre 2020

Pagination : $34-43$

ISSN : 0395-773X

\section{Référence électronique}

Antoine Palévody, "Un théâtre qui traduit ? Pistes de réflexion sur la théâtralité de la traduction »,

Traduire [En ligne], 243 | 2020, mis en ligne le 15 décembre 2020, consulté le 31 décembre 2020. URL: http://journals.openedition.org/traduire/2127 ; DOI : https://doi.org/10.4000/traduire.2127 


\section{Un théâtre qui traduit?}

Pistes de réflexion sur la théâtralité de la traduction

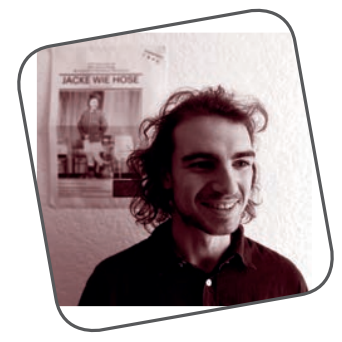

\section{Antoine Palévody}

Comment une pensée de la traduction pourrait-elle mettre au jour une théorie du théâtre? Si, depuis les années 1980, les interrogations quant à la spécificite de la traduction théâtrale se sont multipliées ${ }^{1}$, c'est bien souvent pour énoncer les difficultés de traduction posées par le texte dramatique (principalement liées à la question de l'oralité). Mais à ces réflexions, si justes soient-elles quant à la pratique du traducteur, manque cependant la charge problématique que porte l'adjectif «théâtral». Or réfléchir à ce qui fait la théâtralité de la traduction peut être l'occasion de revoir cette notion pour le moins fuyante. Cela implique d'envisager la traduction théâtrale non pas comme une somme de stratégies imposées par le texte dramatique, mais comme une manière parmi d'autres de faire du théâtre, rejoignant par là l'une

1. Pour ne citer que quelques-unes des plus récentes publications, voir Traduire, 2010, "Traduire pour le théâtre», 222; Frigau Manning Céline et Karsky Marie Nadia (dir.), Traduire le théâtre, Saint-Denis, Presses universitaires de Vincennes, coll. "Théâtres du monde», 2017; Théâtre/ Public, 2020: «Traduire. Carte blanche à la maison Antoine Vitez», 235. 
des thèses fondamentales d'Antoine Vitez pour qui "l'art du théâtre est une affaire de traduction» (Vitez [1991] 2015, 125). Ainsi, deux champs d'investigation réciproques se dégagent: quelle théâtralité s'exprime dans le traduire, d'une part, et qu'est-ce qui, dans les processus de création théâtrale, relève de la traduction? Cela nous invite à mettre la réflexion sur la traduction à l'épreuve des études théâtrales et de la création contemporaine pour établir les rapports qui structurent l'un et l'autre. D'où l'interrogation que nous nous proposons de soulever ici quant à un théâtre qui traduit.

\section{Ce que traduire dit de la théâtralité}

Traduire pour le théâtre est très régulièrement assimilé à un travail sur la corporalité des mots, sur le rythme des répliques, et les traducteurs affirment se focaliser davantage sur les sonorités et la prosodie, sur leurs sens donc, plutôt que sur une supposée signification à retranscrire ${ }^{2}$. Jean-Michel Déprats est ainsi l'un des premiers à avoir mis en valeur cet impératif de la primauté du corps, découvrant chez Shakespeare, qui était lui-même acteur, la dimension "pneumatique ${ }^{3}$ " (Déprats [1996] 2017, 178) du texte dramatique, la marque d'une écriture calibrée pour des "souffles» (Déprats 1982, 45). Si ces thèses sont encore régulièrement citées, c'est qu'elles se justifient par une idée aussi vraie que répandue: celle qui fait du corps - celui de l'acteur qui prononce la traduction ou du traducteur travaillant à partir de son propre corps ${ }^{4}$ - l'instance de vérification de la traduction d'un texte dramatique. Comme le dit Michel Bataillon, "la traduction théâtrale est immédiatement sanctionnée par une personne physique» (Bataillon 1990, 70).

Cela pose cependant un problème majeur, avec le risque de réduire la théâtralité à la seule qualité rythmique du texte. Un premier aspect de ce problème est souligné par JeanLouis Besson, qui signale que la sanction immédiate du texte

2. Voir Palimpsestes, 2016, «Les sens en éveil: traduire pour la scène», et notamment Génin 2016, 14.

3. Il emprunte le terme à Vitez cité par Bataillon in ATLAS 1990, 70.

4. Voir Éloi Recoing, "Poétique de la traduction théâtrale», in Traduire, 222, 2010, p. 103 124. 
par l'acteur laisse peu de place à l'appropriation lente que demande une forme résolument nouvelle, car la traduction tendrait alors "à modéliser les œuvres, à les fondre dans le moule d'une "langue de théâtre" dont personne ne sait vraiment ce qu'elle recouvre» (Besson 2005, 710-711) ${ }^{5}$.

Cependant, une autre charge problématique de l'assimilation de la théâtralité au rythme se dégage à la lumière des travaux d'Henri Meschonnic. Le rythme, selon lui, est "l'organisation du mouvement dans la parole» (Meschonnic [1999] 2012, 69) qui s'exprime par la présence sonore et prosodique d'une subjectivité dans le discours. Fondamentalement oral, le rythme produit la «théâtralité du langage» à l'œuvre dans toute poétique, aussi bien chez Shakespeare ou Kafka, que chez Humbolt $(2012,281)$. Mais cette attention à la théâtralité, dont se réclament de nombreux traducteurs de théâtre, n'est alors paradoxalement plus le propre de la traduction théâtrale puisqu'elle est l'exigence de toute traduction littéraire $(2012,34)$. En d'autres termes, rythme et oralité ne peuvent suffire à distinguer la traduction théâtrale d'un travail littéraire. En effet, selon la théorie de Déprats, ces derniers permettent bien la «mise en bouche» du texte; mais le théâtre ne saurait être réduit à la simple émission d'un texte par des acteurs. Or c'est peut-être dans l'attention à ce que le texte fait non seulement au corps, mais aussi à l'espace où celui-ci évolue que la traduction réalise sa pleine théâtralité, dans une prise en charge dramaturgique, et non pas seulement linguistique, de l'entièreté du devenir scénique d'une œuvre ${ }^{6}$.

Dès lors, dans le contexte théâtral, c'est peut-être la notion de geste qui permet le mieux de compléter la poétique du rythme. En effet, alors que celui-ci exprime un rapport au temps, le geste s'inscrit dans un rapport à l'espace. Craig faisait d'ailleurs du geste l'unité fondamentale du théâtre: "[le geste] est à l'Art du Théâtre ce que le dessin est à la peinture, la mélodie à la musique. L'Art du Théâtre est né du geste - du mouvement - de la danse» (Craig [1911] 1999, 138).

5. Un exemple frappant de ce phénomène serait la traduction de La Movette de Tchékhov par Elsa Triolet. Voir la comparaison très détaillée de cette traduction à celle de Vitez in Meschonnic 2012, 492-526.

6. Voir Pavis 1990, 138-139. 
La différence entre le poète et le dramaturge réside en ce que ce dernier s'adresse aux yeux plus encore qu'aux oreilles, «toujours au moyen du mouvement» (1999, 139). Le geste complète le rythme en ouvrant le traduire à tout ce qui est mouvement sur la scène, non seulement dans les mots (ce qui relève du rythme), mais aussi, littéralement, entre eux (ce qui relève du geste). L'enjeu n'est pas seulement, en effet, de traduire les gestes induits par la parole ${ }^{7}$, mais aussi tous les mouvements dramaturgiques qui constituent un texte: "ll ne s'agit pas d'imiter une œuvre dans une autre langue, mais de saisir, à défaut de l'œuvre tout entière, le (ou les) geste(s) qui la constituent. On ne les saisit bien sûr que fugitivement» (Jourdheuil 1982, 35). Ces gestes ne se révèlent que dans le rapport du texte à la scène, ils ne sont que suggérés par les mots, sans que ces derniers ne les recouvrent complètement, et c'est dans leur élucidation que réside la tâche proprement dramaturgique du traducteur. Le geste met en effet à l'épreuve du traduire tout ce qui, au théâtre, échappe à la communication langagière: l'idéologie par le gestus brechtien ${ }^{8}$, l'éloignement d'une culture par la "traduction intergestuelle ${ }^{9} »$, ou même l'inconscient d'une écriture par la gestuelle désarticulée dont pourrait témoigner un spectacle de Claude Régy.

Traduire les gestes place ainsi le traducteur dans une position intermédiaire entre le texte et la scène, faisant de la théâtralité son principal enjeu. En effet, on peut lire à l'article "Théâtralité» du Lexique du drame moderne et contemporain que «le concept de théâtralité permet d'articuler le théa $\hat{a}^{-}$ tral et le non-théâtral, puisqu'il peut rendre compte d'un désir de théâtre dans ce qui n'en est pas encore, et qu'il éclaire le lien entre texte et représentation» (Sarrazac 2010, 213). La théâtralité n'est donc pas nécessairement contenue dans

7. Voir l'exemple donné par Déprats de la traduction de l'inclinaison du buste induite par le vers I humbly thank you in Déprats [1996] 2017, 176.

8. Pour un exemple de traduction du gestus, voir l'anecdote rapportée par Bataillon in Carré Alice et Métais-Chastanier Barbara, "Où commence la dramaturgie», in Traduire, 222, 2010, p. 92-93.

9. Voir Pavis 1990, 160. 
le texte ${ }^{10}$, elle se détermine au contraire dans le processus de traduction lui-même, qui construit un «désir de théâtre». Ce n'est donc pas la qualification dramatique du texte à traduire qui donne à la traduction son caractère théâtral (il est tout à fait possible de traduire théatralement un texte non dramatique), mais la capacité de celle-ci à engager une définition du théâtre, définition qui évolve en fonction du contexte historique ${ }^{11}$. C'est en cela que la traduction peut être un moyen de renouveler le concept de théâtralité. Aussi, si l'on considère que le geste constitue l'élément théâtral essentiel, la traduction se fait théâtrale dans sa capacité à projeter des gestes dans l'espace.

\section{Présence en scène de la traduction}

Par le geste, le travail sur les mots prend alors un nouveau sens, celui d'une trace éphémère laissée dans l'espace ${ }^{12}$, inscrivant à même la scène la marque du traduire. Mais quelle est la teneur de cette trace? Comment la traduction est-elle rendue visible sur scène? On a souvent insisté sur la faillibilité de la traduction, due au caractère intraduisible de toute œuvre. Mais cette faiblesse du traduire ouvre un potentiel créatif, dès lors que la scène fait le choix d'accueillir les manques de la traduction. Antoine Vitez a ainsi mis en avant la nécessité de «sous-traduire» (Vitez 1982, 9), c'est-à-dire de se contenter d'indiquer une différence (par la syntaxe, par exemple) là où le traducteur est incapable de recréer cette différence. Rester "volontairement en deçà du spectacle ou de l'effet littéraire originel» (Vitez [1991] 2015, 341), montrer le manque, voire le mettre en scène, donne à la traduction

10. Pavis se dit ainsi contre «toute sémiologie théatrale qui présuppose a priori que le texte dramatique possède une théâtralité qu'il s'agit à tout prix d'extirper du texte, pour l'exprimer sur la scène» $(1990,30)$.

11. Meschonnic, qui affirme qu'écrire implique toujours une théorie sur le langage soulignait aussi qu'«un texte et ses traductions sont dans des histoires et des langues différentes, et surtout des stratégies et des enjeux différents» (Meschonnic [1999] 2012, 85).

12. Vitez, qui voyait dans l'éphémère la marque commune du théâtre et de la traduction, dit d'ailleurs que «l'acteur est un poète qui écrit sur le sable» (Vitez [1991] 2015, 144). 
la puissance suggestive d'un écho. Cela renvoie à une manière, pour reprendre les termes de Georges Didi-Huberman, non pas de dire, mais d'

accentuer la vérité. De l'éclairer - fugitivement, lacunairement - par instants de risque, décisions sur fond d'indécisions. De lui donner de l'air et du geste. Puis, de laisser sa place nécessaire à l'ombre qui se referme, au fond qui se retourne, à l'indécision qui est encore une décision de l'air. C'est donc une question, une pratique de rythme: souffle, geste, musicalité. C'est donc une respiration. Accentuer les mots pour faire danser les manques et leur donner puissance, consistance de milieu en mouvement. Accentuer les manques pour faire danser les mots et leur donner puissance, consistance de corps en mouvement ${ }^{13}$.

Le mouvement réciproque des formules de Georges DidiHuberman nous éclaire sur la dialectique du présent et de l'absent à l'œuvre dans la traduction, où le texte original n'apparaît que "lacunairement». Ainsi, en traduisant les Psaumes pour le spectacle Comme un chant de David de Claude Régy (2005), Henri Meschonnic rend sensible cette respiration de ce qui ne peut être traduit que par intermittence. Fidèle à ses recherches sur la "théâtralité du langage», il bouscule la syntaxe et le phrasé français au gré des allitérations et des accents disjonctifs et conjonctifs de l'hébreu, donnant littéralement de l'air et du geste au texte en marquant ces derniers par des blancs sur la page. Le texte traduit garde ainsi la marque des manques qui séparent les Psaumes de leur traduction "délibérément contemporaine» (MervantRoux 2008, 115). Or Claude Régy, pour qui «plus une œuvre est trovée, plus elle semble vouée à la scène car les creux qui l'évident l'animent aussi du souffle de la voix originelle ${ }^{14}$ », s'empare dans sa mise en scène de ce que les manques de la traduction offrent comme «part de silence» $(2008,150)$. L'écart de la traduction devient alors visible jusque dans l'espace scénique où «fondus au noir, silence, lents mouvements

13. Didi-Huberman Georges, Gestes d'air et de pierre, Paris, Éditions de Minuit, 2005, p. 9. 14. Claude Régy remarque lui-même la parenté de sa théorie du théâtre avec la fameuse thèse de la «langue pure» défendue par Walter Benjamin dans «La tâche du traducteur». Voir Jérôme Hankins, Claude Régy, "L'interprète du silence» in Nouvelle revue d'esthétique, 3, 2009, p. 75-81. 
de lumière constituent parfois les stigmates de ce qui a disparu et signalent, en creux, les endroits où le travail de remaniement a entraîné une suppression» $(2008,151)$. La traduction théâtrale se fait alors elle-même geste de mise en scène, celui qui opère le passage du chant de David à comme un chant de David, logeant dans cette identité toute relative le potentiel de création du théâtre. Le geste du traduire entretient dès lors lui aussi une dimension temporelle, bien différente de celle du rythme: celle de l'œuvre du temps. Dessinant derrière lui sa trace dans l'espace, le geste garde le souvenir du chemin parcouru. Le théatre qui prend son parti de la traduction prend dès lors aussi celui de la «nostalgie de l'original» (Jourdheuil 1982, 35) qui est chez Régy une nostalgie de l'origine.

\section{Le théâtre de la traduction}

On voit ainsi que la traduction théâtrale dépasse la question du texte, en ce qu'elle s'inscrit dans un espace qu'elle façonne par le mouvement. II s'agit dès lors de se demander en quoi elle est elle-même un geste théâtral, pour poser la question de ce que serait un théâtre qui traduit. Nous rejoignons en cela la voie ouverte par Antoine Vitez, selon qui le théâtre est fondamentalement acte de traduction. Mise en scène et traduction sont liées selon lui par le fait qu'elles sont autant impossibles que nécessaires. II ne s'agit donc pas seulement de métaphore de la mise en scène comme "traduction intersémiotique», mais d'une affirmation quant à la capacité du théâtre à toujours inventer. En effet, si Antoine Vitez affirme que «c'est bien parce qu'on ne peut pas traduire que la mise en scène est une traduction» (Vitez 1982, 8), c'est parce que «l'irréductibilité du poète» $(1982,7)$ - l'intraduisible - somme la mise en scène d'inventer des gestes nouveaux. De la même manière que c'est le caractère «injouable» d'une œuvre qui pousse le metteur en scène à la création, c'est selon Antoine Vitez «la difficulté du modèle, son opacité, [qui] provoque le traducteur à l'invention dans sa propre langue, l'acteur dans son corps et sa voix» (Vitez [1991] 2015, 125). Mais l'intérêt de cette invention, et ce qui fait qu'elle est proprement «traduction», est qu'elle ne prétend pas être création pure. Au contraire, l'art théâtral, "art de la variation», se distingue pour Antoine Vitez 
par son obligation de toujours «refaire» $(2015,123)$, précisément parce que la traduction parfaite est impossible: "On est convoqué devant le tribunal du monde à traduire, et la traduction est perpétuellement à refaire. Image de l'Art luimême, de l'art théâtral, qui est l'art de la variation infinie. II faut rejouer, toujours rejouer; tout recommencer» $(2015,112)$. Créer de la différence dans la répétition pourrait dès lors être la maxime de ce théâtre fondé sur la traduction. Le théâtre occupe ainsi cette position ambiguë, à la fois «laboratoire des gestes et des paroles de la société » et "conservateur des formes anciennes» $(2015,110)$, un art qui imite tout en créant des effets d'étrangeté, caractéristique de toute traduction véritable $e^{15}$.

La force de la «traduction généralisée» à l'œuvre dans le travail théâtral d'Antoine Vitez est donc moins celle de la quête incessante «d'équivalents possibles» comme le suggèrent dans un premier temps Georges Banu et Danièle Sallenave $(2015,586)$, que l'exploration constante d'une dialectique de l'identité et de l'altérité. Cette exploration n'est d'ailleurs pas dépourvue d'un potentiel de contestation dans sa propension à faire "autre chose que les gestes jugés acceptables et normaux» (Vitez 1982, 8). À peine esquissés les contours de ce que pourrait être un théâtre qui traduit apparaît la nécessité d'en déterminer les enjeux politiques et moraux. Vitez dit d'ailleurs lui-même que "la traduction, pas plus que le théâtre, ne peut être considérée en elle-même. Elle est toujours située dans le champ des forces politiques, elle est l'objet d'un enjeu politique et moral» (Vitez [1991] 2015, 112).

Aussi nous proposons-nous de conclure par l'évocation d'une ultime traduction indispensable à l'activité théâtrale: celle qu'opère chaque spectateur vis-à-vis du spectacle ${ }^{16}$. En effet, dans ses écrits sur les arts vivants, il semble que Jacques Rancière assimile le potentiel émancipateur de l'activité spectatrice à un travail de traduction. II évoque ainsi, à

15. Ayant choisi de jover Phèdre avec des costumes entièrement reconstitués d'après l'époque, Vitez remarque que les acteurs "ressembl[aient] à des monstres étranges», découvrant ainsi que, paradoxalement, "c'est la proximité qui accentue l'étrangeté» (Vitez [1991] 2015, 197-198).

16. Voir Pavis 1990, 138 et son article «Dramaturgie nouvelle» in Horizons/théatre, 6, 2016, p. 105-110. 
propos de la danse, la nécessité d'une "double traduction», l'une sculptant l'espace par le geste, l'autre faisant de cet espace un poème: "celle de la danseuse créant un milieu hors d'elle-même et celle du spectateur qui traduit le texte ou l'un des textes possibles que son mouvement écrit sans paroles» (Rancière 2018, 101). Par cette traduction de l'image en poème, le spectateur s'approprie la représentation sans que lui soit dicté un sens. "Faire danser les manques» prend alors une importance accrue en étendant l'espace de rêverie qui laisse libre le spectateur d'opérer, pour en revenir à Vitez, son propre «choix de la hiérarchie des signes ${ }^{17}$ » (Vitez 1982, 9). Ainsi, la traduction du théâtre, "c'est le pouvoir qu'a chacun ou chacune de traduire à sa manière ce qu'il ou elle perçoit, de le lier à l'aventure intellectuelle singulière qui les rend semblables à tout autre sans pour autant que cette aventure ne ressemble à aucune autre» (Rancière 2008, 23).

\section{antoine.palevody@gmail.com}

Né à Toulouse en 1999, Antoine Palévody poursuit actuellement son cursus d'études théâtrales à l'ENS de Lyon. II a notamment traduit de l'allemand Ce que vit le rhinocéros lorsqu'il regarda de l'autre côté de la clôture de Jens Raschke, avec le soutien de la Maison Antoine Vitez. Il est aussi membre du Comité Collisions - Comité de lecture dramatique d'Occitanie - depuis sa création en 2016.

SOURCES CITÉES

Actes des sixièmes assises de la traduction littéraire, Arles, Actes Sud/Atlas, coll. «Littérature», 1990.

CRAIG Edward Gordon, De l'Art du théâtre, Belval, Circé, coll. «Penser le théâtre», [1911] 1999.

DÉPRATS Jean-Michel, «Traduire Shakespeare pour le théâtre», in Théâtre/Public, 44, 1982, p. 45-48.

17. «Pour moi, traduction ou mise en scène, c'est le même travail, c'est l'art du choix dans la hiérarchie des signes.» 
DÉPRATS Jean-Michel, «Traduire Shakespeare», in BANU Georges, REY François, et al., Antoine Vitez, le devoir de traduire, Arles, Actes Sud, coll. «Apprendre», [1996] 2017, p. 170-178.

GÉNIN Isabelle, «Présentation», in Palimpsestes, 29, 2016, p. 9-20.

JOURDHEUIL Jean, «De quoi parlions-nous?», in Théâtre/Public, 44, 1982, p. 35.

MERVANT-ROUX Marie-Madeleine (dir.), Claude Régy. Les voies de la création théâtrale, Paris, CNRS Éditions, coll. «Arts du spectacle», 2008.

MESCHONNIC Henri, Poétique du traduire, Paris, Verdier, coll. «Verdier/poche», [1999] 2012.

PAVIS Patrice, Le Théâtre au croisement des cultures, Paris, José Corti, coll. «Rien de commun», 1990.

RANCIÈRE Jacques, Le Spectateur émancipé, Paris, La Fabrique éditions, 2008.

RANCIÈRE Jacques, Les Temps modernes, Paris, La Fabrique éditions, 2018.

SARRAZAC Jean-Pierre (dir.), Lexique du drame moderne et contemporain, Belval, Circé, coll. «Penser le théâtre», 2010, p. 213-217.

VITEZ Antoine, «Le devoir de traduire», in Théâtre/Public, 44, 1982, p. 6-9.

VITEZ Antoine, Le Théâtre des idées, anthologie proposée par Danièle Sallenave et Georges Banu, Paris, Gallimard, coll. «Le Messager», [1991] 2015. 